Revue d'histoire de l'Amérique française

\title{
Lettre de J.-J. Girouard à sa femme (16 janvier 1838)
}

Volume 7, numéro 1, juin 1953

URI : https://id.erudit.org/iderudit/301580ar

DOI : https://doi.org/10.7202/301580ar

Aller au sommaire du numéro

\section{Éditeur(s)}

Institut d'histoire de l'Amérique française

\section{ISSN}

0035-2357 (imprimé)

1492-1383 (numérique)

Découvrir la revue

Citer ce document

(1953). Lettre de J.-J. Girouard à sa femme (16 janvier 1838). Revue d'histoire de l'Amérique française, 7(1), 110-112. https://doi.org/10.7202/301580ar d'utilisation que vous pouvez consulter en ligne.

https://apropos.erudit.org/fr/usagers/politique-dutilisation/ 


\title{
DOCUMENTS INÉDITS
}

\author{
(Documents Girouard, suite)*
}

\section{J.-J. GIROUARD A SA FEMME}

Ma chère amie,

Prison de Montréal, 16 janvier 1838.

J'aurais pu t'écrire plus tôt d'une manière furtive mais je n'ai pas voulu enfreindre les instructions données aux geoliers défendant toute communication et j'ai mes raisons pour me soumettre strictement à tous les règlemens et restrictions auxquels l'autorité a juger à propos de soumettre les prisoniers. Cependant je t'ai fait donner de mes nouvelles toutes les fois que j'en ai pu trouver l'occasion et j'ai eu le plaisir d'apprendre qu'au milieu des calamités qu'il a plu à la providence de nous départir tự ne t'étais pas découragée et qu'au reste tu avais trouvé en attendant un gîte chez nos bons amis de Rigaud que tu salueras bien de ma part sans oublier la maison de Monsieur Larocque et bien moins encore Madame Dumouchel si elle se trouve avec toi. Dis à celle-ci que le papa ${ }^{1}$ est bien et qu'il va de mieux en mieux, non qu'il ait été malade mais je l'avais trouvé en arrivant ici dans un état d'abattement qui aurait pu le rendre malade et qui s'est peu à peu dissipé. Hercule et Camille sont très bien et s'amusent à chanter et à jouer à differens jeux avec leurs compagnons de détention qui sont assez joyeux. Il serait trop long, ma bonne amie, de te donner des détails de tout ce qui m'est arrivé depuis mon départ de St-Benoit. Malette ${ }^{2}$ a du te conter ma route jusqu'à l'endroit ou il m'a laissé. $Q u$ 'il te suffise de savoir pour le moment que j'avais choisi une retraite sure où je devais demeurer jusqu'à ce que je pus traverser et me mettre hors d'atteinte du côté de mes ennemis: ce que j'aurais pu effectuer avec facilité en suivant le plan arrêté avec

* Voir Revue d' Histoire de l'A mérique française. (Mars 1953): 564-70.

1. Jean-Baptiste Dumouchel, marchand de Saint-Benoît, incarcéré avec ses deux fils Camille et Hercule.

2 Aucun Mallette ne figure dans les Patriotes de 1897-1838 d'Aegidius Fauteux. Dans son Tableau (encore inédit, croyons-nous), des Patriotes qui ont eté incarcérés dans les prisons de Montréal... Girouard mentionne trois Mallet: Frs Mallet 58 ans, cultivateur de Saint-Eustache, emprisonné le 17 déc. 1837, relâché le 28 fév. 1838; Jos. Mallet, cultivateur, âgé de 21 ans, aussi de Saint-Eustache, arrêté le 14 déc. 1837, relâché le même jour que le précédent; Vital Mallet, 40 ans, cultivateur de la Pointe-Claire, emprisonné le 8 janv. 1838. 
un homme très au fait des routes et sur lequel je pouvais compter. Mais lorsque j'eus appris que tous ou presque tous nos amis s'étaient laissés prendre, que les deux jeunes Masson ${ }^{3} \&$ ensuite $\mathrm{Mr}$ Dumouchel et ses deux fils étaient en prison, j'ai de suite changé ma détermination et j'ai compté que ce serait de ma part une espèce de lâcheté de les abandonner dans une conjoncture aussi pénible, et ou je pouvais leur être utile. Je résolus donc de me rendre avec eux et de partager leur sort quel qu'il fut. Cependant la difficulté était de faire le trajet sans m'exposer aux insolences \& même aux violences de nos ennemis qui gardaient toutes les routes. Avant de laisser St Benoit j'hésitai \& je fus presque tenté d'y demeurer pour tâcher de sauver quelques morceaux du naufrage, et surtout pour obtenir des officiers anglais que les papiers publics que j'avais entre mes mains fussent protégés contre le vendalisme des bandes qui ne respiraient que pillage et désolation. Mais il était un peu tard, et je dus me soustraire aux poignards des incendiaires qui ont parcouru mon malheureux comté. Le jour de Noel donc j'écrivis sans détour mes intentions au colonel Simpson ${ }^{4}$ en lui disant que je n'aurais aucune objection à me remettre entre ses mains, persuadé que j'étais de son honneur et de sa générosité, et qu'avec sa protection je pourrais aller joindre mes amis sans être sujet aux risées, aux infamies \& même aux brutalités auxquels plusieurs patriotes ont été exposés en allant à la prison, et le lendemain, peu après midi j'y étais rendu sans que personne le sut.

N'ayant que peu d'argent \& ne pouvant calculer le terme de ma détention, j'ai refusé de prendre ma pension du concierge qui est très chère: d'ailleurs les morceaux ne pouvaient être que rarement bien conditionnés, vû la difficulté de règler les repas, à cause des fréquentes visites que font ici les autorités, et parce que le Dr Nelson ${ }^{5} \&$ moi nous sommes dans des chambres séparées, et qu'il est défendu que nous communiquions avec les autres. Je te donnerai une autre fois des détails de mon petit ménage. Tout ce que je puis te dire aujourd'hui, e'est que je vis assez bien. M. Bélinge ${ }^{6} \mathrm{~m}^{\prime}$ 'envoie des viandes cuites, et je t'assure que ma gargotte est assez bonne, et que je me porte bien. Je vais passer à quelque chose de plus important; car toi, ma pauvre amie, comment vis-tu?

Tu trouveras ci-inclus une procuration sous seing privé pour Félix, en attendant que je puisse en envoyer une plus formelle pour toi et pour lui;

3. Damien et Luc-Hyacinthe Masson. Voir Les Patriotes de 1887-1838 d'Aegidius Fauteux, p. 318-21 et la Revue d'Histoire de l'Amerique française, III: 349-366, article de Robert-Lionel Séguin.

4. John Simpson, tory, officier de douane à Côteau-du-Lac. Député d'York de 1824 à 1827, puis de Vaudreuil de 1841 à 1844. La veuve épousée par Simpson était mère de John Arthur Roebuck. Par conséquent Roebuck n'était pas le beaupère de Simpson, ainsi que le prétend faussement Aegidius Fauteux (Les Patriotes de 1837-1838, p. 254).

5. Dr Wolfred Nelson, chef des "Patriotes" à Saint-Denis sur Richelieu. Incarcéré le 13 décembre 1837.

6. S'agit-il de Félix-Moïse Bélinge de Montréal, étudiant en droit et.“"fils de la liberté", ou de son père Etienne Belinge, époux de Félicité Dumouchel? 
car j'ai présenté à cet effet une requête au Commandant des forces, Sir John Colborne. Dans la même requête je lui expose aussi l'état des prisonniers et de la prison, je lui demande quelque allouance additionnelle pour la nourriture des prisoniers dénués de moyens et surtout ceux qui ont dernièrement été ruinés; je lui parle de l'état peu salubre de plusieurs galeries de la prison encombrés de détenus, et je demande de son humanité qu'il soit adopté au plutôt des mesures sanitaires pour prévenir des maladies dangereuses que produiraient inévitablement la malpropreté, la mauvaise odeur, le défaut d'air renouvellé, le manque de linge et surtout d'une nourriture assez abondante, \& enfin je supplie que l'on nous accorde, à nous qui ne sommes pas encore juridiquement accusés, et encore bien moins condamnés, les consolations religieuses que l'on accorde sans difficulté dans des prisons qui ne renferment que des criminels subissant leur sentence. En attendant M. Blanchet, curé de St-Charles ${ }^{7}$, notre confrère prisonier, fait régulièrement des prières et des lectures de piété.

Pour revenir à la procuration, je ne te donne non plus qu'à Félix, aucune instruction particulière, bien assuré que vous ferez tout pour le mieux. Qu'il tâche de rassembler mes minutes, répertoires et autres papiers et qu'il les garde en sa possession, aucune autorité n'a droit d'en disposer sans mon consentement, et si ceux qui ont pu s'emparer de quelqu'un de mes papiers se refusent à les lui livrer, j'espère bientôt obtenir de Son Excellence les ordres nécessaires pour les y contraindre. Si parmi mes effets qui n'ont pas été pillés ou brulés vous pouviez trouver mes quatre boites de pastels et le rouleau de papier gris ou roux fait de bourre de soie exprès pour ces crayons, il faudrait m'envoyer cela dans une boîte bien clôse et dans du fourrage. Je m'en amuserais bien ici où j'essayerais à faire quelques portraits. Si Félix a pu mettre la main sur le dictionnaire de Boiste, ${ }^{8}$ je le prie de me l'envoyer.

Ecrivez-moi tous deux bien au long et bien en détail en quel état vous vous trouvez, Ovide, Arthemise \& la famille de M. Barcelo et tous nos bons et vertueux et braves et si dévoués patriotes; quels sont vos moyens d'existence, quels effets ont été sauvés des flammes et du pillage, quel espoir vous pouvez avoir d'en recouvrer quelques débris, \& c. Le frère de notre Snowdon est venu me voir et m'a montré beaucoup de sensibilité. Il m'a promis ses services et m'a même dit qu'il vous verrait.

Sans doute, ma tendre amie, lorsque tout ce que nous possédions sur la terre nous a été enlevé, tu as fait comme moi au bon Dieu la prière de Job... et ainsi que moi tu as étê consolée. Courage donc, confiance en la providence, et surtout résignation entière et sans réserve. Embrasse les tous pour ton affectionné,

\section{J.-J. Girouard}

7. L'abbé Augustin-Magloire Blanchet, futur évêque de Nesquallay, Etat de Washington, E.-U. Sur les circonstances de l'emprisonnement de l'abbé Blanchet (16 déc. 183731 mars 1838), voir Notre Mâ̂tre le Passé, II: 107, 112-115.

8. Boiste (Pierre-Claude-Victoire) 1765-1824, lexicographe, auteur d'un Dictionnaire universel de la langue française. 